\title{
EDITORIAL
}

\section{Modulation of cellular behavior by exogenous messenger RNA}

Leukemia (2006) 20, 767-769. doi:10.1038/sj.leu.2404219

A major part of current gene therapy research requires stable integration and long-term expression of a therapeutic gene in order to be effective. ${ }^{1}$ Many preclinical and some clinical efforts try to restore a defective gene function by means of stable gene transfer in diseases like hemophilia, muscular dystrophy, sickle cell anemia and various types of severe combined immunodeficiency. Despite promising preclinical results in various animal models, some human clinical applications have been put on hold owing to unexpected adverse effects related to uncontrolled stable integration of therapeutic genes; ${ }^{2}$ this has led to the search for more safe gene targeting strategies. Next to those applications that require stable transgene expression, there are potential cellular therapies based on immunomodulation or stem cell culture and differentiation that are also in search of clinically applicable and efficient gene transfer techniques. ${ }^{3,4}$ In those circumstances, gene transfer may only need to be transient until the target cell has been instructed what to do. The field of transient over- or neo-expression of specific proteins in specific cell types has been - and still is - dominated by the use of DNAbased vectors. The least efficient method, although in widespread use at least in the laboratory, is based on transient introduction of plasmid DNA into cells by lipofection or electroporation. Although a gene transfer efficiency of $10-50 \%$ using plasmid DNA is considered to be relatively high, in no way it can compete with currently existing viral transduction strategies. Although both DNA and RNA viruses are continuously modified and improved for research applications, specific safety issues, including potential immunogenic and tumorigenic hazards, still need to be carefully addressed before widespread clinical applications.

Parallel to the development of DNA-based gene transfer strategies for transient protein expression, the use of mRNA as vector for gene transfer has come of age only relatively recently. The earliest reports describing the usefulness of mRNA transfer were about mRNA microinjection in oocytes. ${ }^{5}$ Apart from this time-consuming, but very useful, single cell-based application, the use of mRNA for gene transfer has been limited owing to the generally accepted idea that mRNA is very unstable, difficult to handle and insufficient to be followed by high levels of protein expression in transfected cells as compared to viral vectors. All these points can now be addressed, because novel technologies have been developed that make mRNA transfection a highly efficient alternative to DNA transfection and very competitive with viral transduction techniques. In 1996, the group of Eli Gilboa opened a new perspective on the use of mRNA for gene transfer in dendritic cells (DC). Dendritic cells are professional antigen-presenting cells of the immune system capable of priming memory and naive $\mathrm{T}$ cells in vitro and in vivo. Their initial studies demonstrated that murine and human DC, passively pulsed or lipofected with defined or total tumor mRNA, were able to induce antigen-specific immune responses. ${ }^{6,7}$ Although lipofection of mRNA coding for the enhanced green fluorescent protein (EGFP) resulted in detectable EGFP expression by flow cytometric analysis, passive pulsing did not result in any detectable EGFP protein. Never- theless, despite the absence of detectable protein expression after passive pulsing of tumor antigen-encoding $\mathrm{mRNA}$, in these and following publications clear biological effects, that is, antigen presentation followed by T-cell activation, have been demonstrated, indicating (1) that mechanisms exist by which cells can take up and translate small amounts of exogenous mRNA and (2) that the transfer of small amounts of exogenous mRNA can indeed modulate the cellular behavior of DC. Although these effects are observed in vitro, it remains to be determined whether they also occur naturally in vivo. In the following years, based on the above-described results, various research groups have developed improved methodologies for in vitro transfer of mRNA into cells. By now, RNA transfer by means of electroporation has become one of the most promising gene transfer applications where short-term introduction of certain proteins is needed in order to obtain a biological effect. For example, (1) DC electroporated with mRNA encoding tumor-associated or viral antigens or co-stimulatory molecules appear to have high T-cell immune stimulatory effects in vitro, ${ }^{8-13}$ (2) unconditioned T cells electroporated with mRNA encoding cloned antigen-specific T-cell receptors appear to be functionally reprogrammed in order to destroy specific target cells; ${ }^{14}$ and (3) stem cell populations electroporated with mRNA encoding specific growth factors appear to become committed towards a specific lineage of differentiation. ${ }^{15,16}$ Altogether, these data support the hypothesis that cellular behavior can be modified by means of in vitro RNA transfer.

A natural in vivo process of RNA crosstalk has recently been demonstrated in plants. Plasmodesmata are plant-specific intercellular organelles that connect the cytoplasm of neighboring plant cells, allowing passive or active exchange of cytoplasmic macromolecules, including proteins and RNA molecules. These plasmodesmata also connect plant cells to the phloem and xylem, the plant long-distance circulatory system that allows transport of water and minerals from roots to leaves (through xylem) and sugars and amino acids from leaves to roots (through phloem). Experimental evidence recently showed that both specific mRNAs and specific small singlestranded RNA molecules are transported throughout the plant body via the phloem. ${ }^{17,18}$ Although still under intensive investigation, this long-distance transmission of RNA molecules via the phloem is believed to be one of the mechanisms used by plants to exert systemic control over developmental, physiological and physiopathological processes, including transposon activity, transcriptional and translational processes and viral infection. ${ }^{19}$ These observations demonstrate that there is not only an in vitro use for mRNA transfer in modulation of cellular behavior, but that an in vivo specific mechanism based on transfer of mRNA has also evolved in order to control cellular behavior.

Although animal cells also have intercellular bridges, these gap junctions have a maximal functional pore size of about $1.5 \mathrm{~nm}$, implying that coupled cells can share their small molecules (such as inorganic ions, sugars, amino acids, nucleotides, vitamins and the intracellular mediators cyclic AMP and inositol trisphosphate) but not their macromolecules (proteins, nucleic acids and polysaccharides). In the field of animal cell signalling, in addition to growth factors, cytokines, 
adhesion molecules and small mediators such as nucleotides, the existence and potential function of cell-derived microvesicles as mediators in cell-to-cell communication has gained much interest. ${ }^{20,21}$ These microvesicles appear to originate from the membrane of activated or apoptotic cells and transfer membrane proteins and RNA towards neighboring cells in an in vitro and presumably in vivo mechanism of cell-to-cell signalling, as demonstrated for the interaction between tumorderived microvesicles and monocytes. ${ }^{22}$ In this issue of Leukemia, Ratajczak et al. ${ }^{23}$ report on the characterization of microvesicles derived from embryonic stem (ES) cells. Analysis of their content, as compared to that of ES cells, revealed that these microvesicles were significantly enriched for specific proteins and protein-encoding mRNAs involved in self-renewal of stem cells. Furthermore, these microvesicles can be used in vitro as physiological liposomes for transfecting immature hematopoietic progenitor cells. Upon release of their content - either protein or translatable mRNA - into hematopoietic progenitors, some degree of increased self-renewal was observed in vitro and in vivo.

Although it is not clear at the moment how such a microvesicle-mediated mRNA-based cell-to-cell communication system is actually influencing cellular behavior in vivo, a recent study describing cytotoxic T-lymphocyte induction after vaccination of mice with liposome-encapsulated mRNA demonstrates the potential power of this novel cell communication tool. ${ }^{24}$ The findings of Ratajczak et al. open many new research perspectives for the development of RNA-based gene transfer strategies in vitro and in vivo. First, as cell-derived microvesicles seem to be acting as physiological liposomes, their potential use, both in vitro and in vivo, might provide an advantage over currently used lipofection or electroporation methodology for transferring exogenous mRNA into cells in order to control and/ or direct cellular behavior. Secondly, the fact that these microvesicles are specifically enriched for certain proteins and transcription factors implies the existence of cellular control mechanisms, possibly unknown targeting signals on protein and mRNA, able to collect and encapsulate these factors into microvesicles. Understanding this mechanism might in time allow for the production of engineered microvesicles containing your favorite proteins or mRNAs that can be used as efficient vehicles for in vitro and in vivo protein or mRNA delivery in animal cells.

\section{Acknowledgements}

The research work of the authors is supported by grant nos. G.0456.03, G.0313.01, 7.0004.03N and WO.012.02 of the Fund for Scientific Research - Flanders (FWO - Vlaanderen), the Foundation against Cancer (Stichting tegen Kanker), the Fund for Cell Therapy from the Antwerp University Hospital and the Concerted Research Action (GOA Fa20000/802) from the University of Antwerp.

\section{P Ponsaerts ${ }^{1,2}$ and ZN Berneman ${ }^{1,2}$ \\ ${ }^{1}$ Laboratory of Experimental Hematology, Faculty of Medicine, Antwerp University, Antwerp, Belgium and ${ }^{2}$ Center for Cell Therapy and Regenerative Medicine, Antwerp University Hospital (UZA), Wilrijkstraat, Edegem, Belgium} E-mail: zwi.berneman@uza.be

\section{References}

1 Van Tendeloo VFI, Van Broeckhoven C, Berneman ZN. Gene therapy: principles and applications to hematopoietic cells. Leukemia 2001; 15: 523-544.
2 Gonin P, Buchholz CJ, Pallardy M, Mezzina M. Gene therapy biosafety: scientific and regulatory issues. Gene Therapy 2005; 12: S146-S152.

3 Breckpot K, Heirman C, Neyns B, Thielemans K. Exploiting dendritic cells for cancer immunotherapy: genetic modification of dendritic cells. J Gene Med 2004; 6: 1175-1188.

4 Menendez P, Wang L, Bhatia M. Genetic manipulation of human embryonic stem cells: a system to study early human development and potential therapeutic applications. Curr Gene Ther 2005; 5: 375-385.

5 Zehavi-Willner T, Lane C. Subcellular compartmentation of albumin and globin made in oocytes under the direction of injected messenger RNA. Cell 1977; 11: 683-693.

6 Boczkowski D, Nair SK, Snyder D, Gilboa E. Dendritic cells pulsed with RNA are potent antigen-presenting cells in vitro and in vivo. $J$ Exp Med 1996; 184: 465-472.

7 Nair SK, Boczkowski D, Morse M, Cumming RI, Lyerly HK, Gilboa E. Induction of primary carcinoembryonic antigen (CEA)-specific cytotoxic T lymphocytes in vitro using human dendritic cells transfected with RNA. Nat Biotechnol 1998; 16: 364-369.

8 Van Tendeloo VF, Ponsaerts P, Lardon F, Nijs G, Lenjou M, Van Broeckhoven $C$ et al. Highly efficient gene delivery by mRNA electroporation in human hematopoietic cells: superiority to lipofection and passive pulsing of mRNA and to electroporation of plasmid cDNA for tumor antigen loading of dendritic cells. Blood 2001; 98: 49-56.

9 Saeboe-Larssen S, Fossberg E, Gaudernack G. mRNA-based electrotransfection of human dendritic cells and induction of cytotoxic T lymphocyte responses against the telomerase catalytic subunit (hTERT). J Immunol Methods 2002; 259: 191-203.

10 Milazzo C, Reichardt VL, Muller MR, Grunebach F, Brossart P. Induction of myeloma-specific cytotoxic $\mathrm{T}$ cells using dendritic cells transfected with tumor-derived RNA. Blood 2003; 101: 977 982.

11 Van Gulck ERA, Ponsaerts P, Heyndrickx L, Vereecken K, Moerman F, De Roo A et al. Efficient stimulation of HIV-1-specific $\mathrm{T}$ cells using dendritic cells electroporated with mRNA encoding autologous HIV-1 Gag and Env proteins. Blood 2006; 107: 18181827.

12 Kavanagh DG, Kaufmann DE, Sunderji S, Frahm N, Le Gall S, Boczkowski D et al. Expansion of HIV-specific CD4 ${ }^{+}$and $\mathrm{CD}^{+}{ }^{+} \mathrm{T}$ cells by dendritic cells transfected with mRNA encoding cytoplasm- or lysosome-targeted Nef. Blood 2006; 107: 1963-1969.

13 Van Driessche A, Gao L, Stauss HJ, Ponsaerts P, Van Bockstaele $\mathrm{DR}$, Berneman ZN et al. Antigen-specific cellular immunotherapy of leukemia. Leukemia 2005; 19: 1863-1871.

14 Schaft N, Dorrie J, Muller I, Beck V, Baumann S, Schunder T et al. A new way to generate cytolytic tumor-specific $T$ cells: electroporation of RNA coding for a T cell receptor into T lymphocytes. Cancer Immunol Immunother 2006; doi: 10.1007/s00262-0050098-2.

15 Van Driessche A, Ponsaerts P, Van Bockstaele DR, Van Tendeloo VF, Berneman ZN. Messenger RNA electroporation: an efficient tool in immunotherapy and stem cell research. Folia Histochem Cytobiol 2005; 43: 213-216.

16 Smits E, Ponsaerts P, Lenjou M, Nijs G, Van Bockstaele DR, Berneman ZN et al. RNA-based gene transfer for adult stem cells and T cells. Leukemia 2004; 18: 1898-1902.

17 Yoo BC, Kragler F, Varkonyi-Gasic E, Haywood V, Archer-Evans S, Lee YM et al. A systemic small RNA signaling system in plants. Plant Cell 2004; 16: 1979-2000.

18 Lucas WJ, Lee JY. Plasmodesmata as a supracellular control network in plants. Nat Rev Mol Cell Biol 2004; 5: 712-726.

19 Haywood V, Yu TS, Huang NC, Lucas WJ. Phloem long-distance trafficking of gibberellic acid-insensitive RNA regulates leaf development. Plant J 2005; 42: 49-68.

20 Heijnen HF, Schiel AE, Fijnheer R, Geuze HJ, Sixma JJ. Activated platelets release two types of membrane vesicles: microvesicles by surface shedding and exosomes derived from exocytosis of multivesicular bodies and alpha-granules. Blood 1999; 94: 3791-3799.

21 Baj-Krzyworzeka M, Majka M, Pratico D, Ratajczak J, Vilaire G, Kijowski J et al. Platelet-derived microparticles stimulate proliferation, survival, adhesion, and chemotaxis of hematopoietic cells. Exp Hematol 2002; 30: 450-459. 
22 Baj-Krzyworzeka M, Szatanek R, Weglarczyk K, Baran J, Urbanowicz B, Branski $\mathrm{P}$ et al. Tumour-derived microvesicles carry several surface determinants and mRNA of tumour cells and transfer some of these determinants to monocytes. Cancer Immunol Immunother 2006; DOI: 10.1007/s00262-005-0075-9.

23 Ratajczak J, Miekus K, Kucia M, Zhang J, Reca R, Dvorak P et al. Embryonic stem cell-derived microvesicles reprogram hemato- poietic progenitors: evidence for horizontal transfer of mRNA and protein delivery. Leukemia 2006; 20: 847-856.

24 Hess PR, Boczkowski D, Nair SK, Snyder D, Gilboa E. Vaccination with mRNAs encoding tumor-associated antigens and granulocyte-macrophage colony-stimulating factor efficiently primes CTL responses, but is insufficient to overcome tolerance to a model tumor/self antigen. Cancer Immunol Immunother 2005; 20: 1-12. 